\title{
Digitale Barrierefreiheit
}

18. September 2019 | TYPO3 University Days 2019

Stefan Farnetani, mindscreen $\mathrm{GmbH}$ 
Digitalstrategie

\section{mindscreen.}

München | Berlin
Digitale Barrierefreiheit

Nutzerorientierte Gestaltung

CMS- und Web-Entwicklung
四 2x BIENE Award 国 TYPO3 Award

国 Neos Award 


\section{Wer ist Stefan Farnetani?}

- Frontend-Entwickler

- Barrierefreiheitsexperte mit über 15 Jahren Erfahrung

- Leiter des Arbeitskreises Barrierefreiheit der German UPA

- Geladener Experte zur Weiterentwicklung des BIK BITV-Tests 
Warum ist

Barrierefreiheit wichtig? 


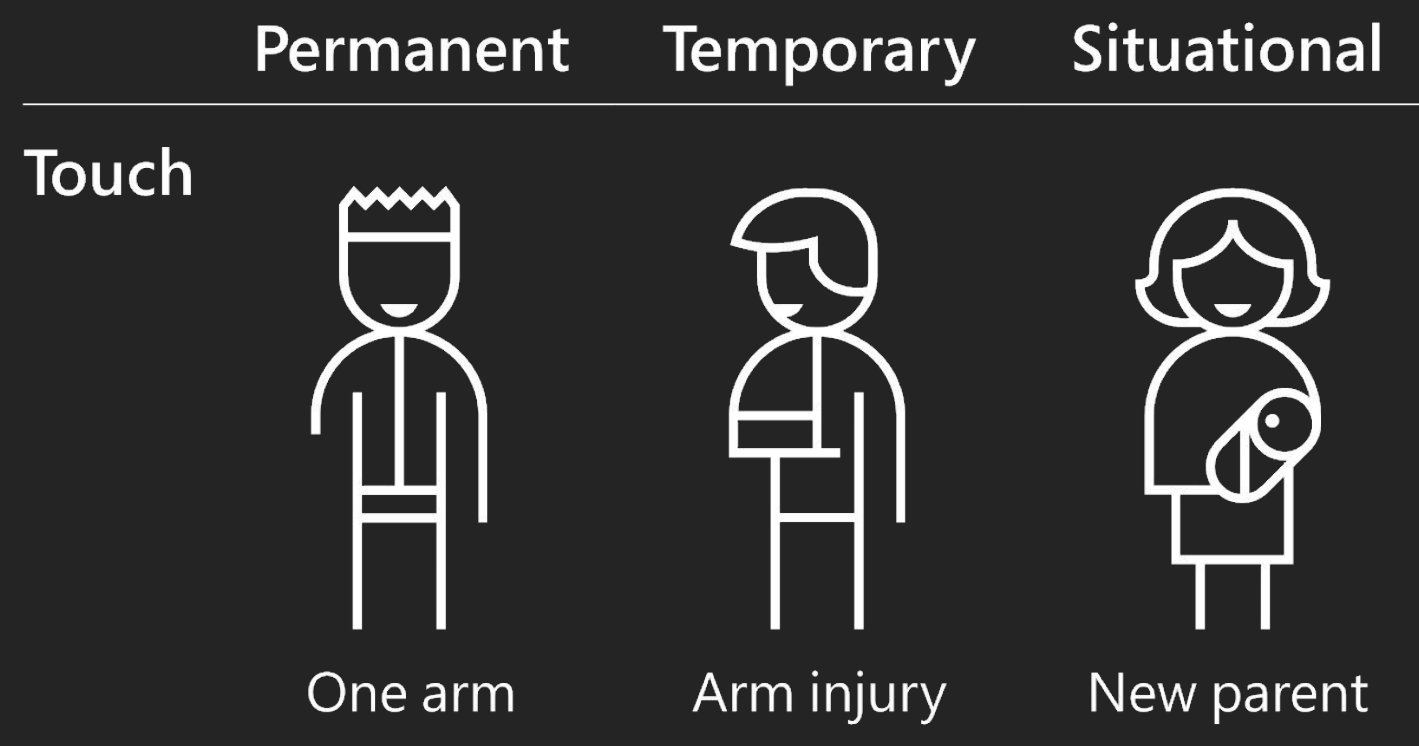

Inclusive Design | Microsoft 

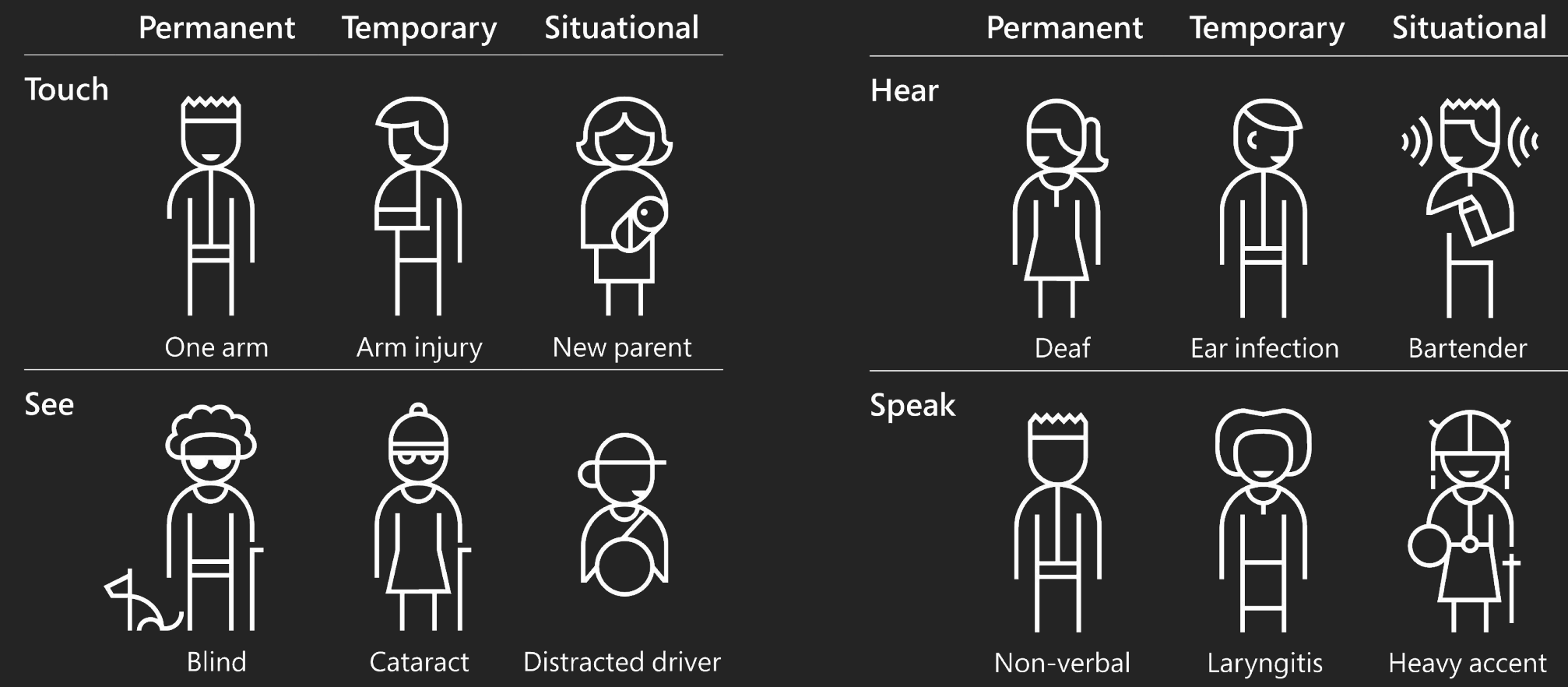

Inclusive Design | Microsoft 


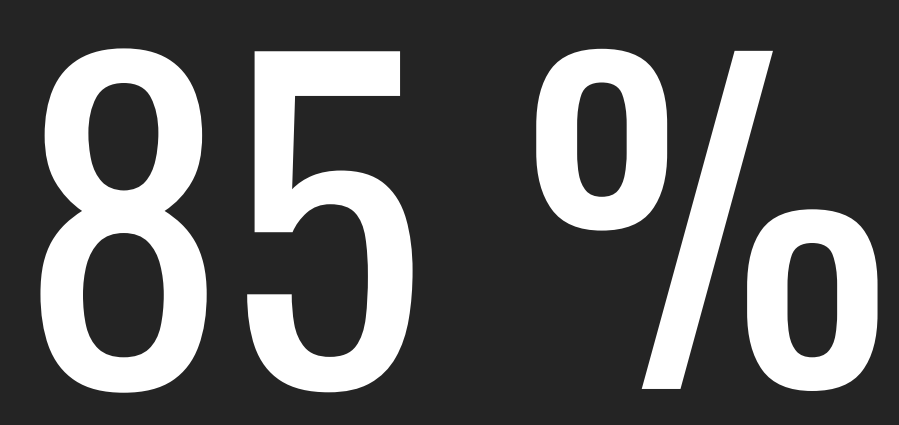

der Facebook-Videos

werden ohne Ton geschaut

Digidav UK 


\section{uns alle! Wir machen es für-etie.}


Gesetze \& Richtlinien

EU Richtlinie 2016/2102 
EU Richtlinie 2016/2102 - für öffentliche Stellen

Ist die Richtlinie über den barrierefreien Zugang zu den Websites und mobilen Anwendungen öffentlicher Stellen

\section{EU Richtlinie 2016/2102}

= Harmonisierung der Webstandards zur Barrierefreiheit in Europa

\section{Mitgliedsländer überführen die Richtlinie in nationales Recht}




\section{EU Richtlinie 2016/2102 - für öffentliche Stellen}

Was

- Websites

- Mobile Anwendungen

- Dateiformate von Büroanwendungen

\section{Bis wann}

- Websites, die ab dem 23. September 2018 veröffentlicht wurden, müssen ab dem 23. September 2019 barrierefrei sein.

- Alle Websites müssen ab dem 23. September 2020 barrierefrei sein.

- Mobile Anwendungen müssen ab dem 23. Juni 2021 barrierefrei sein.

- Ab 23. September 2018: neu veröffentlichte Dateiformate von Büroanwendungen müssen barrierefrei sein (z.B. PDFs).
Weitere Maßnahmen

Erklärung zur Barrierefreiheit
Feedback-Mechanismus
Monitoring und Berichterstattung 
Barrierefreiheit integrieren 
Barrierefreiheit Integrieren

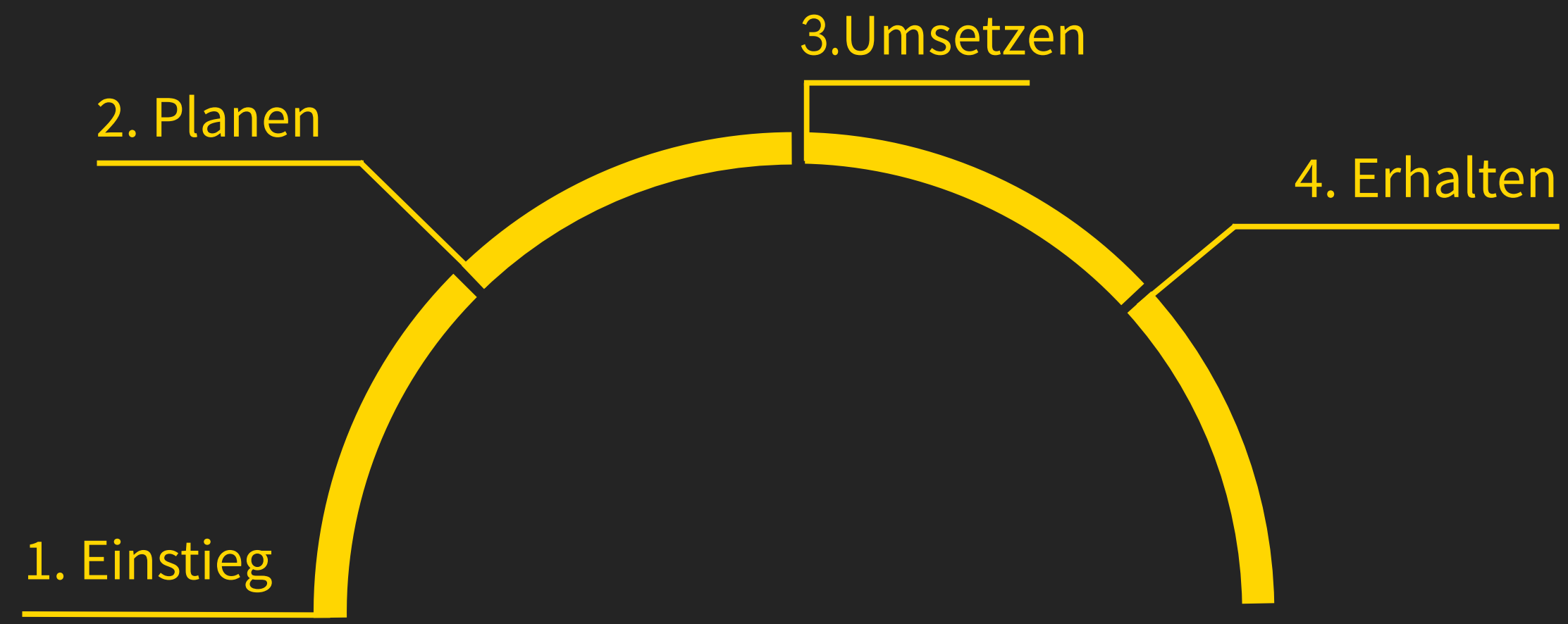

Systematik basiert auf den WAI Planning and Managing web Accessibility 


\section{Barrierefreiheit integrieren}

\section{Einstieg}

- Grundlagen lernen (Schulungen, Workshops)

- Situation, Organisationsstruktur, Infrastruktur erkunden

- Unterstützung suchen

- Ziele festlegen 


\section{Barrierefreiheit integrieren}

\section{Planen}

- Verantwortlichkeiten festlegen

- Status feststellen (Audit, Test, Herstellerauskunft)

- Budget und Ressourcen bestimmen

- Zwischenschritte / messbare Fortschritte festlegen 


\section{Barrierefreiheit integrieren}

\section{Umsetzen}

- Wissen aufbauen, verteilen, erhalten

- Ausschreibungen \& Stellenbeschreibungen prüfen

- Loslegen!

- Aufgaben priorisieren: Hohe Wirksamkeit, leicht zu lösen. 


\section{Barrierefreiheit integrieren}

\section{Erhalten}

- Nutzerfeedback sammeln \& umsetzen

- Neue Technologien, Richtlinien \& Gesetze im Blick behalten

- Monitoring 


\section{Barrierefreiheit \& TYPO3}


Barrierefreiheit ist Teamwork

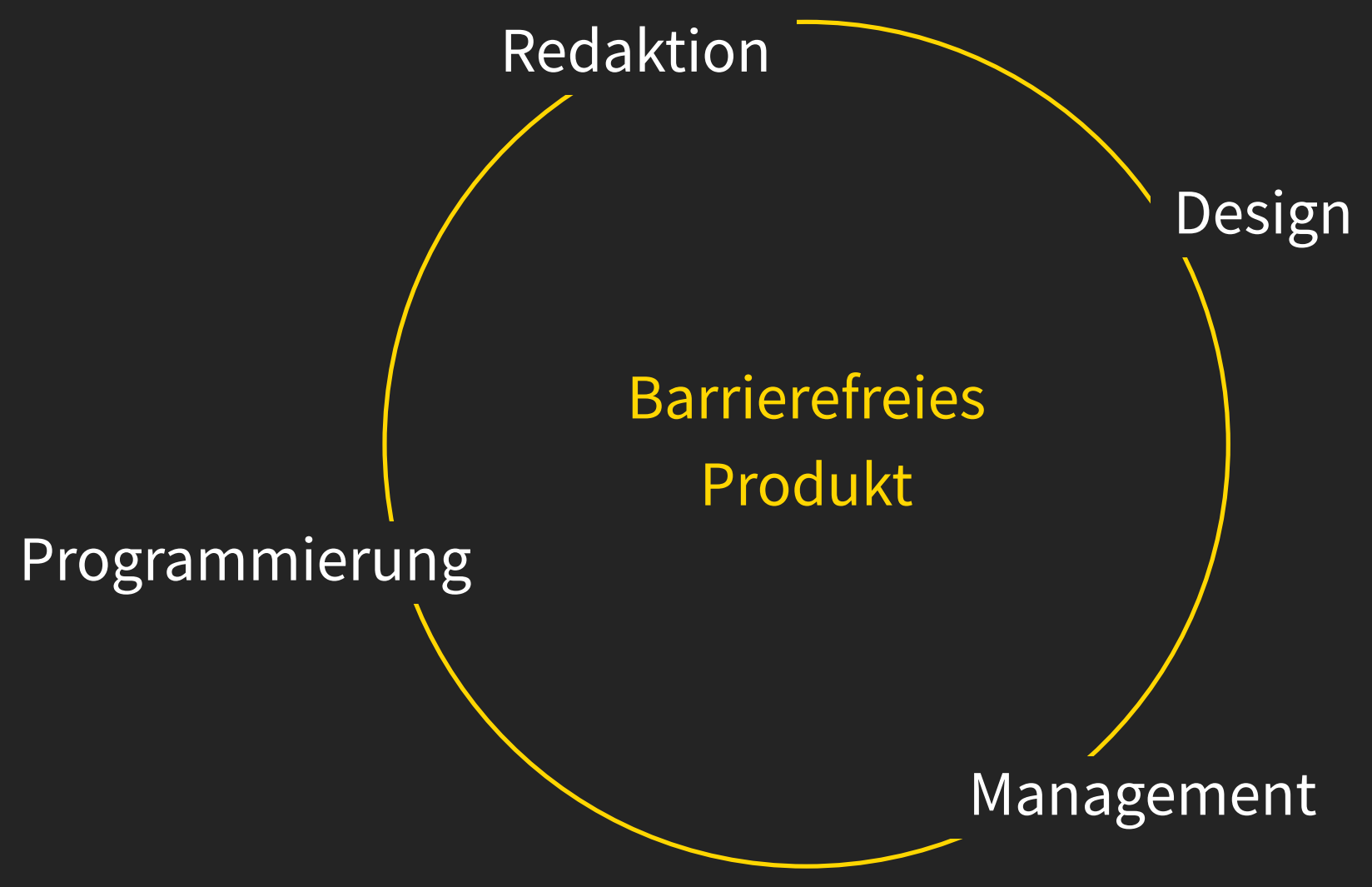


Barrierefreiheit in TYPO3

Ausliefern/Rendern einer barrierefreien

Website

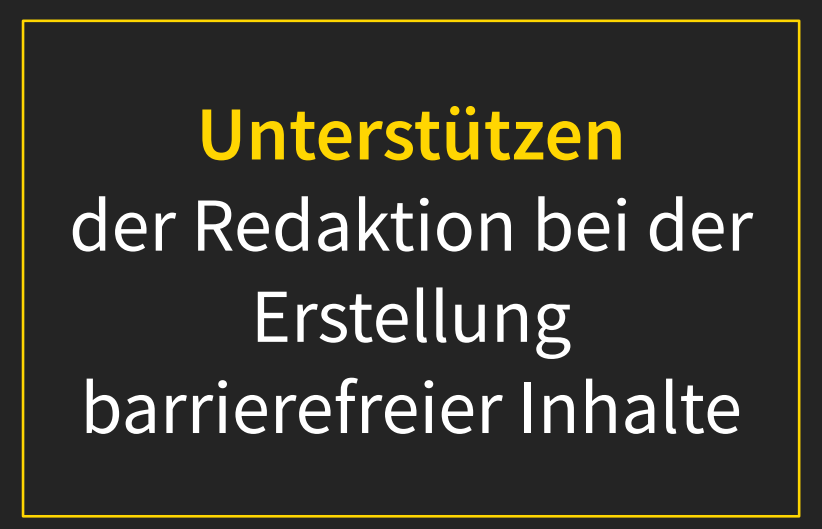

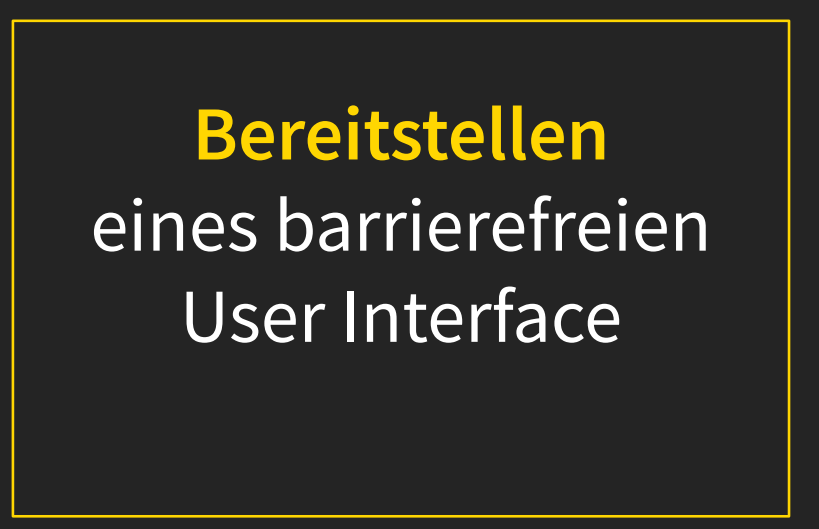


Barrierefreiheit in TYPO3

Ausliefern/Rendern einer barrierefreien Website

\section{Unterstützen} der Redaktion bei der Erstellung barrierefreier Inhalte

\section{Bereitstellen} eines barrierefreien User Interface 
Barrierefreiheit in TYPO3

Ausliefern/Rendern einer barrierefreien Website

\section{Unterstützen} der Redaktion bei der Erstellung barrierefreier Inhalte

\section{Bereitstellen} eines barrierefreien User Interface 
Barrierefreiheit in TYPO3

Ausliefern/Rendern einer barrierefreien

Website

\section{Unterstützen} der Redaktion bei der Erstellung barrierefreier Inhalte

\section{Bereitstellen} eines barrierefreien User Interface 


\section{Wiederbelebung der \\ Accessibility Initiative}




\section{Accessibility Initiative}

\section{Summary}

---- Restarted in September 2019 ---

Raise awareness about accessibility. Provide tools which will help everybody, involved in building websites with TYPO3, to make websites fully accessible. Have an accessible TYPO3 backend.

\section{Background}

Accessibility is an essential part of an inclusive user experience. When you commit to accessibility, you ensure that your product (whether it is a website, web application, or mobile app) is operable by people with a wide range of abilities. As a result, potential barriers to access are not only removed, but you avoid creating them in the first place. No matter if your organization is in the public, private, educational or charity sector, making sure your product is accessible will increase your audience reach.

\section{Get involved!}

- Get in touch via Slack:

\#cig-accessibility

\section{Team}

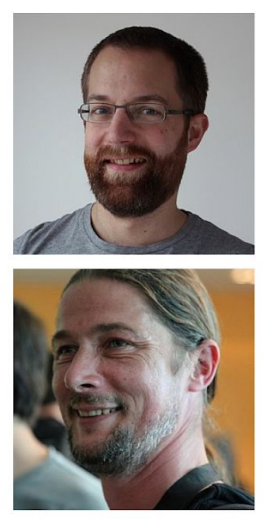

Michael Telgkamp

(Lead)

\section{Patrick Broens}

(Dev, Research) 


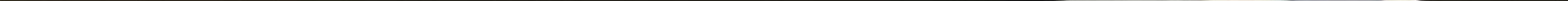




\section{farnetani@mindscreen.de}

Twitter:@ilCapitanoHook

Barrierefreie Webentwicklung

Berater für digitale Barrierefreiheit

mindscreen $\mathrm{GmbH}$

Umsetzung, Beratung, Workshops 